\title{
Removal of Amoxicillin and Sulfanomide by Freshwater Bacteria in Sri Lanka
}

\author{
G.Y. Liyanage, P.M. Manage* \\ Department of Zoology, University of Sri Jayewardenepura, Sri Lanka \\ *pathmalalmanage@yahoo.com
}

\begin{abstract}
Antibiotics are an important group of pharmaceuticals used in human and animal health care. Most of the antibiotics are prone to release to the environment due to improper usage. This has resulted in bacterial resistance development and toxicity in aquatic communities. Present study reports the biodegradation of amoxicillin (AMX) and sulfanomide (SUF) by Bacillus cereus, Enterobacter ludwigii and Enterobacter sp. strains which were previously reported as crude oil degraders. Different concentrations of AMX and SUF (0, 60, 120, 180, 240, 300, $360,420 \mathrm{ppm}$ ) were used to detect minimum inhibition concentration (MIC) by standard pour plate method. AMX and SUF degradation kinetics were studied by introducing $0.5 \mathrm{ml}$ of overnight starved bacterial suspensions into sterile antibiotic medium in triplicate at final concentration of 60 and $120 \mathrm{ppm}$ respectively. The samples were incubated at $28^{0} \mathrm{C}$ shaking at $100 \mathrm{rpm}$ and $0.5 \mathrm{ml}$ sub-sample aliquots were removed at two days interval for a period of 14 days. Analyses of antibiotics were performed by high performance liquid chromatography (HPLC). The MIC values for SUF and AMX were detected as 240, $420 \mathrm{ppm}$ for B. cereus, 120, $360 \mathrm{ppm}$ for E. ludwigii and 180 and $300 \mathrm{ppm}$ for Enterococcus sp. respectively. After 14 days of incubation complete removal of AMX and $80 \%$ degradation of SUF was recorded by bacterium B. cereus. E. ludwigii showed $75 \%$ degradation of AMX and $60 \%$ degradation of SUF where Enterobacter sp. showed degradation of both AMX (80\%) and SUF (70\%) respectively. Thus, the present study illustrate antibiotics degradation potential of microbial community is important to understand their role in removal of antibiotics from the natural environment.
\end{abstract}

Keywords: Biodegradation, Minimum Inhibitory Concentrations (MICs), Amoxicillin, Sulfanomide, Bacillus cereus, Enterobacter ludwigii, Enterobacter sp. 